\title{
Rapid Solution of Integral Equations of Scattering Theory in Two Dimensions
}

\author{
V. ROKHLIN* \\ Department of Computer Science, Yale University, \\ New Haven, Connecticut 06520
}

Received May 23, 1986; revised March 22, 1989

\begin{abstract}
The present paper describes an algorithm for rapid solution of boundary value problems for the Helmholtz equation in two dimensions based on iteratively solving integral equations of scattering theory. CPU time requirements of previously published algorithms of this type are of the order $n^{2}$, where $n$ is the number of nodes in the discretization of the boundary of the scatterer. The CPU time requirements of the algorithm of the present paper are $n^{4: 3}$, and can be further reduced, making it considerably more practical for large scale problems. 1990 Academic Press, Inc.
\end{abstract}

\section{INTRODUCTION}

One of standard approaches to numerical treatment of boundary value problems for elliptic partial differential equations (PDEs) calls for converting them into second kind integral equations (SKIEs) with subsequent discretization of the latter via appropriate quadrature formulae. Discretization of the resulting SKIEs usually leads to dense large-scale systems of linear algebraic equations, which are in turn solved by means of some iterative technique, such as a generalized conjugate residual algorithm (see $[11,23]$ ). Most iterative schemes for the solution of linear systems of this type require application of the matrix of the system to a sequence of recursively generated vectors. Applying a dense matrix to a vector is an order $n^{2}$ procedure, where $n$ is the dimension of the matrix, which in this case is equal to the number of nodes in the discretization of the domain of the integral equation. As a result, the whole process is at least of the order $n^{2}$, and for many large scale problems, this estimate is prohibitively large.

In the present paper, we describe an algorithm for rapid application of matrices resulting from discretization of integral equations of scattering theory in two dimensions to arbitrary vectors. The algorithm requires an amount of work proportional to $n^{4 / 3}$, where $n$ is the number of nodes in the discretization of the boundary of the scatterer, and when it is combined with a generalized conjugate residual-type

* The author was supported in part by the Office of Naval Research under Grant N00014-83-K-0184. 
algorithm, the resulting process takes very few iterations to converge, leading to an order $n^{4,3}$ algorithm for the solution of the original scattering problem.

Reduction of boundary value problems for elliptic PDEs to second kind integral equations is discussed in detail in $[6,16,19]$. Numerical treatment of SKIEs in the general case can be found, for example, in [3], and numerical solution of acoustic scattering problems in two dimensions by means of SKIEs is discussed in [18]. We present an algorithm for rapid solution of integral equations of classical potential theory (Laplace's equation) in [20], and the algorithm of the present paper can be viewed as an extension of the approach of [20] to the case of the Heimholtz equation. However, the analytical apparatus of the present paper is considerably more complicated than the analytical apparatus of [20], reflecting the difference between the behavior of solutions of the Helmholtz equation and that of harmonic functions.

Remark 1.1. While the algorithm of the present paper has an asymptotic CPU time estimate $n^{4,3}$, it can be easily modified into an order $n \log (n)$ algorithra (see Subsection 4.4). However, it appears that this modification would not lead to significant improvement in actual calculation times for most problems of practicable size $(n \leqslant 20,000)$.

The layout of the paper is as follows. In Section 2, we introduce the necessary notation and summarize the facts from mathematics and numerical analysis to be used in the rest of the paper. In Section 3, we develop the mathematical apparasus of this paper (predominantly, functional analytic in nature), used in Section 4 to design an algorithm for the rapid evaluation of integral operators of scattering theory. In Section 5, the implementation of the algorithm is discussed and results of several numerical experiments are presented. Finally, Section 6 discusses ways in which the scheme can be generalized.

\section{BACKGROUND INFORMATION}

\subsection{Notation}

We will be considering the situation depicted in Fig. 1. A fluid scatterer of arbitrary shape is embedded in a two-dimensional fluid space. The boundary of the scatterer parametrized by its length will be denoted by $\gamma$ so that $\gamma:[0, L] \rightarrow \mathbb{R}^{2}$ is a Jordan curve, and the image of $\gamma$ will be denoted by $I$. The open interior of $\gamma$ will be denoted by $\Omega$, so that $\Gamma=\partial \bar{\Omega}$. We will assume that $\gamma$ is at least $c^{2}$, i.e., than $a^{*}$ each point it has at least two continuous derivatives. The interior normal to $y$ at the point $x=\gamma(t)$ will be denoted by $N(t)$, and it will always be assumed that $\|N(t)\|=1$. The density of the scatterer will be denoted by $\rho^{\text {in }}$, and the speed of sound in it will be denoted by $c^{\text {in }}$. The density of the containing space will be denoted by $\rho^{\text {out }}$, and the speed of sound in it will be denoted by $c^{\text {out }}$. We will denote the angular frequency of the source by $\omega$, and its location by $x_{s}$. Finally, will 
denote the Helmholtz coefficients inside and outside the scatterer by $k_{\text {in }}$ and $k_{\text {out }}$, respectively (as is well known, $k_{\text {in }}=\omega / c^{\text {in }}, k_{\text {out }}=\omega / c^{\text {out }}$ ). In the notation introduced above, we assume that $\rho^{\text {in }}$ and $\rho^{\text {out }}$ are positive real numbers, and that $c^{\text {in }}, c^{\text {out }}$, and $\omega$ are complex numbers such that $\operatorname{Re}\left(c^{\text {in }}\right)>0, \operatorname{Re}\left(c^{\text {out }}\right)>0, \operatorname{Re}(\omega)>0$ and $\operatorname{Im}(\omega) \geqslant 0, \operatorname{Im}\left(c^{\text {in }}\right) \leqslant 0, \operatorname{Im}\left(c^{\text {out }}\right) \leqslant 0$.

\subsection{Single and Double Layer Potentials}

For a Helmholtz equation

$$
\nabla^{2} \phi+k^{2} \phi=0,
$$

we will define the field $\phi_{x_{0}}^{k}: R^{2} \backslash\left\{x_{0}\right\} \rightarrow C^{1}$ of a unit charge located at the point $x_{0} \in R^{2}$ by the formula

$$
\phi_{x_{0}}^{k}(x)=H_{0}\left(k\left\|x-x_{0}\right\|\right),
$$

where $H_{0}$ denotes the Hankel function of order zero. We will define the field $\phi_{x_{0}, h}^{k}$ of a unity dipole located at $x_{0}$ and oriented in the direction $h \in R^{2}$ by the formula

$$
x_{x_{0}, h}^{k}(x)=-H_{1}\left(k\left\|x-x_{0}\right\|\right) \cdot \frac{k\left(x-x_{0}, h\right)}{\left\|x-x_{0}\right\|} .
$$

For a continuous function $\sigma:[0, L] \rightarrow C^{1}$, the potential of a single layer of density $\sigma$ on a curve $\gamma$ is a mapping $P_{k \sigma}^{0}: R^{2} \rightarrow C^{1}$ defined by the formula

$$
P_{k \sigma}^{0}(x)=\int_{0}^{L} \phi_{\gamma(t)}^{k}(x) \sigma(t) d t
$$

and the potential of a double layer of density $\sigma$ on a curve $\gamma$ is a mapping $P_{k \sigma}^{1}: R^{2} \rightarrow C^{1}$ defined by the formula

$$
P_{k \sigma}^{1}(x)=\int_{0}^{L} \phi_{\gamma(t), N(t)}^{k}(x) \sigma(t) d t
$$

Remark 2.1. Note that while both $P_{k \sigma}^{0}$ and $P_{k \sigma}^{1}$ are defined on all of $R^{2}$, neither $P_{k \sigma}^{1}$ nor the derivatives of $P_{k \sigma}^{0}$ are continuous in the neighborhood of $\Gamma$. The exact nature of their singularities is crucial for the derivation of Eqs. (2.13), (2.14) of the following subsection, and it is discussed in great detail in $[18,16]$.

\subsection{Acoustic Scattering in Two Dimensions}

In the present paper, we will be considering the following problem:

For a pair of continuous functions $f, g: \Gamma \rightarrow C^{1}$, find two mappings $\phi: \bar{\Omega} \rightarrow C^{1}$, $\psi: R^{2} \backslash \Omega \rightarrow C^{1}$ such that
a.

$$
\nabla^{2} \phi+k_{\text {in }}^{2} \phi=0 \quad \text { on } \Omega
$$
b.

$$
\nabla^{2} \psi+k_{\text {out }}^{2} \psi=0 \quad \text { on } R^{2} \backslash \bar{\Omega}
$$


c.

$$
\begin{array}{rlrl}
\rho^{\text {in }} \cdot \phi-\rho^{\text {out }} \cdot \psi & =f & \text { on } \Gamma \\
\frac{\partial}{\partial N}(\phi-\psi) & =g & & \text { on } \Gamma
\end{array}
$$

e. $\psi$ satisfies the radiation condition at $\infty$, i.e., for any $x \in R^{2}$, there exists $c \in C^{1}$ such that

$$
\lim _{t \rightarrow x_{0}} \psi(t \cdot x) \cdot e^{-i k_{t} \cdot|x|} \cdot \sqrt{(\hat{\imath})}=c
$$

with $k=k_{\text {out }}$.

The above five equations describe acoustic scattering from a two-dimensional fluid inclusion in a fluid space in the frequency domain and have been studied in great detail (see, for example, $[2,10,17,18,5]$ ). Their numerical solution, however, presents a number of serious difficulties, especially for large-scale problems. Here we will follow the approach of [18], which calls for reducing these equations to second kind integral equations and solving the latter numerically via the Nystrom algorithm (see [3]). As is shown in $[17,18]$, by introducing two new unknown functions $\sigma, \eta:[0, L] \rightarrow C^{1}$ and representing the functions $\phi, \psi$ in the form

$$
\begin{aligned}
& \phi=\frac{1}{\rho^{\text {in }}} \cdot P_{k_{\text {tn }}}^{0} \eta+P_{k_{\text {, n }}}^{1} \sigma \\
& \psi=\frac{1}{\rho^{\text {out }}} \cdot P_{k_{\text {out }}}^{0} \eta+P_{k_{\text {out }}}^{1} \sigma,
\end{aligned}
$$

Eqs. (2.1)-(2.5) are reduced to a pair of second kind integral equations on the boundary of the scatterer,

$$
\begin{aligned}
& -2 i\left(\rho^{\text {out }}+\rho^{\text {in }}\right) \sigma+\left(P_{k_{\text {cut } \eta}}^{0}-P_{k_{\text {in }}}^{0}\right)+\left(\rho^{\text {out }} P_{k_{\text {out }} \sigma}^{1}-\rho^{\text {in }} \rho_{k_{\mathrm{In}} \sigma}^{1}\right)=\hat{j}, \\
& 2 i\left(\frac{1}{\rho^{\text {out }}}+\frac{1}{\rho^{\text {in }}}\right) \eta+\frac{\partial}{\partial N}\left(P_{k_{\text {nut }} \sigma}^{1}-P_{k_{\text {in }} \sigma}^{\mathrm{i}}\right) \\
& +\frac{\partial}{\partial N}\left(\frac{1}{\rho^{\text {out }}} P_{k_{\text {ou } \eta}}^{0}-\frac{1}{\rho^{\text {in }}} P_{k_{\mathrm{n} n} \eta}^{0}\right)=g .
\end{aligned}
$$

\subsection{Iterative Solution Of Second Kind Integral Equations}

The system of equations (2.13), (2.14) satisfies the conditions of the Fredholm theorems and can be efficiently solved by means of generalized conjugate residualtype iterative algorithms (see $[11,18,23])$ ). Iterative solution of integral equations usually involves application of the integral operator in the ieft-hand side of the equation to a sequence of recursively generated functions. Applying an integral 
operator to a function numerically is, generally speaking, an order $n^{2}$ procedure, where $n$ is the number of nodes in the discretization of the domain of the operator. The resulting CPU time estimate for the solution of the original scattering problem is also of the order $n^{2}$ (see $[3,18]$ ), which can be prohibitively expensive for largescale problems. The rest of this paper is devoted to constructing an algorithm for numerically applying the integral operators in the left-hand side of Eqs. (2.13), (2.14) to arbitrary functions in a "fast" manner, i.e., for a cost less than $n^{2}$ (the particular algorithm we have tested has an asymptotic CPU time estimate $n^{4 / 3}$ ).

Remark 2.2. Evaluating integral operators in the left-hand sides of Eqs. (2.13), (2.14) numerically can be viewed as evaluating the fields and normal derivatives of the fields created on the curve $\gamma$ by charge and dipole distributions on that same curve. In the following section, we develop an analytical apparatus for rapid evaluation of fields (and derivatives of the fields) of distributions of charges and dipoles, and in Section 4, we use this apparatus to construct an actual algorithm for rapid evaluation of integral operators of the forms (2.13), (2.14).

\subsection{Asymptotic Behavior of Bessel Functions}

In agreement with standard practice, we will denote by $J_{m}$ the Bessel function of the first kind of order $m$, and by $H_{m}$, the Hankel function of order $m$. As is well known (see, for example, [22]) $J_{m}$ are analytic on the whole complex plane for all values of $m$ while $H_{m}$ have a branch cut along the negative real axis and become infinite at the origin. The asymptotic behavior of the functions $J_{m}, H_{m}$ for large $m$ is given by the formulae

$$
\lim _{m \rightarrow \infty} J_{m}(z) \cdot\left(\frac{2 m}{e z}\right)^{m} \cdot \sqrt{(2 \pi m)}=1
$$

and

$$
\lim _{m \rightarrow \infty} H_{m}(z) \cdot\left(\frac{e z}{2 m}\right)^{m} \cdot \frac{\sqrt{(\pi m)}}{\sqrt{2}}=-1
$$

(see $[1,9.3 .1,9.3 .2,9.1 .3]$ ). For large $z$ and fixed $m$, the asymptotic behavior of $J_{m}(z), H_{m}(z)$ is given by the formulae

$$
\begin{aligned}
& \sqrt{z} J_{m}(z)-\sqrt{\left(\frac{2}{\pi}\right)} \cos \left(z-\frac{m \pi}{2}-\frac{\pi}{4}\right)=O\left(\frac{e^{\operatorname{Im}(z)}}{|z|}\right) \\
& \sqrt{z} H_{m}(z)-\sqrt{\left(\frac{2}{\pi}\right)} e^{i(z-m \pi i 2-\pi ; 4)}=O\left(\frac{e^{-\operatorname{Im}(z)}}{|z|}\right)
\end{aligned}
$$

when $z \rightarrow \infty$, as long as $\operatorname{Im}(z) \geqslant 0$ (see $[1,9.2 .5,9.2 .7]$ ). 


\section{Rapid Evaluation of Radiation Fields}

\subsection{Partial Wave Expansions of Radiation Fields}

If a function $\phi: R^{2} \rightarrow C^{1}$ satisfies the Helmholtz equation (2.1) in an open disk $D$ of radius $R$ with the center at the point $x_{0} \in R^{2}$ then there exists a unique sequence $\alpha=\left\{\alpha_{m}\right\}, m=0, \pm 1, \pm 2, \ldots$ such that for any $x \in D$,

$$
\phi(x)=\sum_{m=-\infty}^{+\infty} \alpha_{m} J_{m}(k \rho) e^{i m \theta} .
$$

In the above formula, $\rho=\left\|x-x_{0}\right\|$ and $\theta$ is the angle between the vector $x-x_{0}$ and the $x$ axis.

If a function $\psi$ satisfies Eq. (2.1) outside $D$ and the radiation condition (2.10) at $x$, then there exists a unique sequence $\beta=\left\{\beta_{m}\right\}, m=0, \pm 1, \pm 2, \ldots$ such that for any $x \in R^{2}: \bar{D}$,

$$
\psi(x)=\sum_{m=-\infty}^{+\infty} \beta_{m} H_{m}(k \rho) e^{i m \theta} .
$$

A derivation of the formulae (3.1), (3.2) can be found, for example, in [16], and we will refer to functions satisfying the Helmholtz equation as radiation fields, to expansions of forms (3.1), (3.2) as $J$-expansions and $H$-expansions, respectively, and to the point $x_{0}$ as the center of the expansions (3.1), (3.2).

The following lemma is a direct consequence of the formulae $(2.15),(2.16)$. It establishes the convergence rates of the expansions (3.1), (3.2).

LEMMA 3.1. If $D_{1} \subset D$ is a disk of radius $R_{1}<R$ with the center at $x_{0}$ then there exists $c>0$ such that for any $x \in D_{1}$ and $N>|\dot{k}| \cdot R_{\mathrm{i}}$,

$$
\left|\phi(x)-\sum_{m=-N}^{N} \alpha_{m} J_{m}(k \rho) e^{i m z \theta}\right|<c\left(\frac{R_{1}}{R}\right)^{N} .
$$

If $D_{2} \supset D$ is a disk of radius $R_{2}>R$ with the center at $x_{0}$ then there exists $c>0$ such that for any $x \in R^{2}: \bar{D}_{2}$ and $N>|k| \cdot R$,

$$
\left|\psi(x)-\sum_{m=-N}^{N} \beta_{m} H_{m}(k \rho) e^{i m \theta}\right|<c\left(\frac{R}{R_{2}}\right)^{N} .
$$

Remark 3.1. In numerical calculations, expansions (3.1), (3.2) are truncated after a finite number of terms, and the resulting expressions are viewed as approximations to the dields $\phi, \psi$. If we want to approximate $\phi$ by an expansion of the form (3.3) with an accuracy $\varepsilon$ then according to the above lemma, we have to choose

$$
N \geqslant \max \left(|k| \cdot R_{1}, \frac{-\ln (\varepsilon)+\operatorname{in}(c)}{\ln (R)-\ln \left(R_{1}\right)}\right)
$$


Since logarithm is a very slowly growing function, for medium and large scale problems,

$$
\max \left(R_{1} \cdot|k|, \frac{-\ln (\varepsilon)+\ln (c)}{\ln (R)-\ln \left(R_{1}\right)}\right) \sim R_{1} \cdot|k| ;
$$

i.e., the number of terms in the approximation is almost independent of $\varepsilon$ and must be roughly equal to $|k| R_{1}$. A similar calculation shows that for medium to large scale problems, the expansion (3.2) can be truncated after approximately $N \geqslant|k| R$ terms.

\subsection{Translation Operators for $H$ and $J$ Expansions}

For a real number $r>0$, we will denote by $X_{r}$ the linear space of all two-sided complex sequences $\alpha=\left\{\alpha_{m}\right\}, m=0, \pm 1, \pm 2, \ldots$ such that for some $c>0$,

$$
\left|\alpha_{m}\right| \cdot\left(\frac{2 m}{e r}\right)^{m} \cdot \sqrt{m}<c
$$

for all $m \geqslant r$. We will denote by $Y_{r}$ the linear space of all complex sequences $\beta=\left\{\beta_{m}\right\}, m=0, \pm 1, \pm 2, \ldots$, such that for some $c>0$,

$$
\left|\beta_{m}\right| \cdot\left(\frac{e r}{2 m}\right)^{m} \cdot \sqrt{m}<c
$$

for all $m \geqslant r$. It is easy to see that $X_{r} \subset Y_{r}$, and that the condition (3.6) is a very restrictive one, since in order to satisfy it, the elements of the sequence $\left\{\alpha_{m}\right\}$ must decay as $(r / 2)^{m} / m$ !, while the condition (3.7) is a very mild one-it prohibits the elements of $\left\{\beta_{m}\right\}$ from growing faster than approximately $(2 / r)^{m} \cdot m$ !. By applying formulae (9.3.1), (9.3.2) from [1], it is easy to show that in (3.1). (3.2), $\alpha \in Y_{|k| R}$ and $\beta \in Y_{|k| R}$. Conversely, for any sequence $\alpha \in Y_{|k| R}$, the expansion (3.1) converges inside $D$, and for any $\beta \in Y_{|k| R}$, the expansion (3.2) converges outside $D$. For a natural $n$, we will denote by $T_{n}$ a linear mapping $Y_{r} \rightarrow Y_{r}$ converting a sequence $\alpha=\left\{\alpha_{m}\right\}, m=0, \pm 1, \pm 2, \ldots$ into a sequence $\tilde{\alpha}=\left\{\tilde{\alpha}_{m}\right\}, m=0, \pm 1, \pm 2, \ldots$ defined by the formulae

$$
\begin{array}{lll}
\tilde{\alpha}_{m}=\alpha_{m} & \text { for } & |m| \leqslant n \\
\tilde{\alpha}_{m}=0 & \text { for } & |m| \geqslant n+1 .
\end{array}
$$

Clearly, $T_{n}\left(Y_{r}\right) \subset X_{r}$, and for obvious reasons, we will refer to $T_{n}$ as truncation.

For the remainder of this section, $D_{1}, D_{2}, D_{3}$ will denote three disks in $R^{2}$ such that $D_{2} \subset D_{1}$ and $D_{1} \cap D_{3}=\varnothing$ (see Fig. 1a). The centers and radii of these disks will be denoted by $c_{1}, c_{2}, c_{3}$ and $R_{1}, R_{2}, R_{3}$, respectively. We will denote the distance $\left\|c_{2}-c_{1}\right\|$ by $\rho_{12}$, and the distance $\left\|c_{3}-c_{1}\right\|$ by $\rho_{13}$. The angle between the vector $c_{2}-c_{1}$ and the $x$ axis will be denoted by $\theta_{12}$, and the angle between $c_{3}-c_{1}$ and the $x$ axis will be denoted by $\theta_{13}$. For a point $x \in R^{2}$, we will denote $\left\|x-c_{1}\right\|$ 
$\rho^{\text {out }}: c^{\text {cut }}$

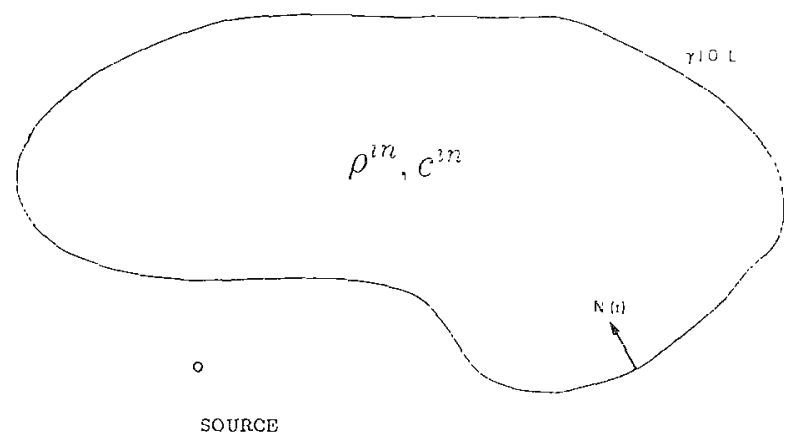

FiG. 1. A fluid scatterer imbedded in a two-dimensional fluic space.

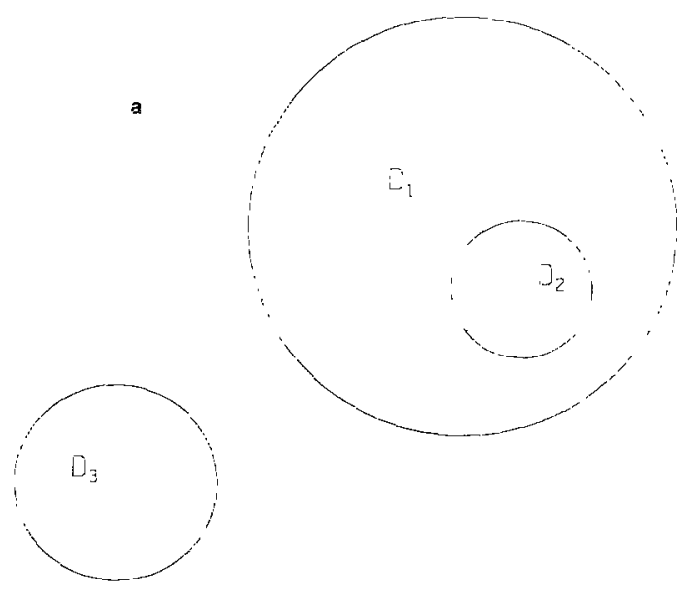

Fig. 1a. Three disks in the plane (Section 3).

by $\rho_{1},\left\|x-c_{2}\right\|$ by $\rho_{2}$, and $\left\|x-c_{3}\right\|$ by $\rho_{3}$. Finally, the angles between the vectors $x-c_{1}, x-c_{2}$, and $x-c_{3}$ and the $x$ axis will be denoted by $\theta_{1}, \theta_{2}, \theta_{3}$, respectively.

Suppose that $n$ is a natural number or $\infty$. We will define a linear operator $U_{c_{2} c_{i}}^{n}: X_{|k| R_{2}} \rightarrow X_{|k| R_{1}}$ as follows. If $A=\left\{a_{m}\right\}, m=0, \pm 1, \pm 2, \ldots$ is an element of $X_{|k| R_{2}}$ then $U_{c_{2} c_{1}}^{n}(A)=B=\left\{b_{m}\right\}, m=0, \pm 1, \pm 2, \ldots$ is defined by the formula

$$
b_{m}=\sum_{j=-n}^{n} e^{i j \theta_{12}} a_{m-j} J_{j}\left(k \rho_{12}\right)
$$

for all $m=0, \pm 1, \pm 2, \ldots$. An operator $V_{c_{1} \kappa_{2}}^{n}: Y_{\mid k_{i} R_{1}} \rightarrow Y_{|k| R_{2}}$ will be defined by the formula

$$
b_{m}=\sum_{j=-n}^{n} e^{-i j\left(\theta_{12}-\pi\right\rangle} a_{m-j} J_{j}\left(k \rho_{12}\right)
$$


for any $A=\left\{a_{m}\right\}, B=\left\{b_{m}\right\}$ such that $B=V_{c_{1} c_{2}}^{n}(A)$. Finally, the operator $X_{|k| R_{3}} \rightarrow Y_{|k| R_{3}}$ converting an arbitrary sequence $A=\left\{a_{m}\right\} \in X_{|k| R_{1}}$ into the sequence $B=\left\{b_{m}\right\} \in Y_{|k| R_{3}}$ defined by the formula

$$
b_{m}=\sum_{j=-n}^{n} e^{-i j\left(\theta_{13}-\pi\right)} a_{m-j} H_{j}\left(k \rho_{23}\right)
$$

will be denoted by $W_{c_{1} c_{3}}^{n}$. For any natural $m$, we will define the operators $U_{c_{2} c_{1}}^{n m}: X_{|k| R_{2}} \rightarrow X_{|k| R_{t}}, V_{c_{1} c_{2}}^{n n}: Y_{|k| R_{1}} \rightarrow Y_{|k| R_{2}}, W_{c_{1} c_{3}}^{n m}: X_{|k| R_{1}}$ by the formulae

$$
\begin{aligned}
U_{c_{2} c_{1}}^{n m} & =T_{m} \cdot U_{c_{2} c_{1}}^{n} \cdot T_{m}, \\
V_{c_{1} c_{2}}^{n m} & =T_{m} \cdot V_{c_{1} c_{2}}^{n} \cdot T_{m}, \\
W_{c_{1} c_{3}}^{n m} & =T_{m} \cdot W_{c_{1} C_{3}}^{n} \cdot T_{m} .
\end{aligned}
$$

Remark 3.2. We will denote by $Y$ the set of all two-sided complex sequences $\left\{y_{i}\right\}, i= \pm 1, \pm 2, \ldots$. When $n$ is a natural number (as opposed to $\infty$ ), the formulae (3.8), (3.9), (3.10) define mappings $\widetilde{U}_{c_{2} \mathrm{C}_{1}}^{n}, \widetilde{V}_{c_{1} \mathrm{C}_{2}}^{n}, \widetilde{W}_{c_{1} c_{3}}^{n}: Y \rightarrow Y$ that can be viewed as extensions of the mappings $U_{c_{2} c_{1}}^{n}, V_{c_{2} c_{2}}^{n}, W_{c_{1} c_{3}}^{n}$ i.e.,

$$
\tilde{U}_{c_{2} c_{1} \mid X_{|k| R_{2}}}^{n}=U_{c_{2} C_{1}}^{n}, \quad \tilde{V}_{c_{1} C_{2} \mid X_{|k| R_{1}}}^{n}=V_{c_{2} C_{1}}^{n}, \quad \tilde{W}_{c_{1}\left[3 \mid X_{|k| R_{1}}\right.}^{n}=W_{c_{1 C_{3}}}^{n} .
$$

Whenever there is no danger of confusion, we will make no distinction between the mappings $\widetilde{U}_{c_{2} c_{1}}^{n}, \widetilde{V}_{c_{1} C_{2}}^{n}, \widetilde{W}_{c_{1} c_{3}}^{n}$ and the mappings $U_{c_{2} c_{1}}^{n}, V_{c_{1} c_{2}}^{n}, W_{c_{1} c_{3}}^{n}$, respectively.

The following three lemmas justify our referring to the operators $U_{c_{2} C_{1}}^{n}, V_{\mathrm{C}_{1} C_{2}}^{n}$, $W_{c_{1} c_{3}}^{n}, U_{c_{2} c_{1}}^{n m}, V_{c_{1} c_{2}}^{n m}, W_{c_{1} c_{3}}^{n m}$ as translation operators, and will be used to shift the origins of $H$ and $J$ expansions, and to convert $H$-expansions into $J$-expansions. They are a direct consequence of the Graf's addition theorem for Bessel functions (see $[1,(9.1 .79)])$.

LEMMA 3.2. Suppose that $\psi: R^{2} \rightarrow C^{1}$ is a radiation field analytical in $R^{2} \backslash \bar{D}_{2}$ and satisfying the radiation condition $(2.10)$ at $\propto$. Suppose further that $\psi$ is represented by an expansion

$$
\gamma(x)=\sum_{m=-\infty}^{+\infty} \beta_{m} H_{m}\left(k \rho_{2}\right) e^{i m \theta_{2}}
$$

valid in $R^{2} \backslash \bar{D}_{2}$, and by an expansion

$$
\psi(x)=\sum_{m=-\infty}^{+\infty} \tilde{\beta}_{m} H_{m}\left(k \rho_{1}\right) e^{i m \theta_{1}}
$$

valid in $R^{2} \backslash \bar{D}_{1}$. Then $\left\{\bar{\beta}_{m}\right\}=U_{c_{2} c_{1}}^{\infty}\left(\left\{\beta_{m}\right\}\right)$. 
LEMMA 3.3. Suppose that $\phi: R^{2} \rightarrow C^{1}$ is a radiation field analytical in $D_{1}$ and represented by an expansion

$$
\phi(x)=\sum_{m=-\infty}^{+\infty} \alpha_{m} I_{m}\left(k \rho_{i}\right) e^{i m \theta_{1}}
$$

valid in $D_{i}$, and by an expansion

$$
\phi(x)=\sum_{m=-\infty}^{+\infty} \tilde{x}_{m} J_{m}\left(k \rho_{2}\right) e^{i m \theta_{2}}
$$

ralid in $D_{2}$. Then $\left\{\tilde{\alpha}_{m}\right\}=V_{c_{1} c_{2}}^{x}\left(\left\{\alpha_{m}\right\}\right)$.

LEMMA 3.4. Suppose that $\psi: R^{2} \rightarrow C^{1}$ is a radiation field analytical outside the disk $D_{1}$ and satisfying the radiation condition $(2.10)$, and that it is represented in $R^{2} \backslash \bar{D}_{1}$ by the expansion (3.12). Then inside the disk $D_{3}$, the function $\psi$ can be represented in the form

$$
\psi(x)=\sum_{m=-\infty}^{+\infty} \gamma_{m} J_{m}\left(k \rho_{13}\right) e^{l m \theta_{3}}
$$

$w i \hat{i} h\left\{\gamma_{m}\right\}=W_{c_{1} c_{2}}^{x}\left(\left\{\tilde{\beta}_{m}\right\}\right)$.

Under the conditions of Lemma 3.2, we will define a radiation field $d^{n}$; $R^{2}: \bar{D}_{1} \rightarrow C^{1}$ by the expression

$$
\psi_{c_{2} c_{1}}^{n}(x)=\sum_{m=-x}^{+\infty} \delta_{m} H_{m}\left(k \rho_{1}\right) e^{i m \theta_{1}}
$$

with the coefficients $\left\{\delta_{m}\right\}, m=0, \pm 1, \pm 2, \ldots$ defined by the formula $\left\{\delta_{m}\right\}=$ $U_{c_{2} c_{1}}^{n}\left(\left\{\beta_{m}\right\}\right)$. Similarly, under the conditions of Lemma 3.3, we will define a radiation field $\psi_{c_{1} C_{2}}^{n}: D_{2} \rightarrow C^{1}$ by the formula

$$
\phi_{c_{1} c_{2}}^{n}(x)=\sum_{m=-x}^{+\infty} \tilde{\delta}_{m} J_{m}\left(k \rho_{2}\right) e^{i m \theta_{2}}
$$

with $\left\{\tilde{\delta}_{n n}\right\}=V_{c_{1} c_{2}}^{n}\left(\left\{\alpha_{m}\right\}\right)$. Finally, under the conditions of Lemma 3.4 , we will denote by $\psi_{c_{2} c_{3}}^{n}$ the radiation field $D_{3} \rightarrow C^{1}$ defined by the formula

$$
\Psi_{c_{2} c_{3}}^{n}(x)=\sum_{m=-\infty}^{+\infty} \bar{\delta}_{m} J_{m}\left(k \rho_{3}\right) e^{l m \theta_{3}}
$$

with the sequence $\left\{\delta_{m}\right\}, m=0, \pm 1, \pm 2, \ldots$ defined by the formula $\left\{\delta_{n_{i}}\right\}=$ $W_{c_{1} !}^{n}\left(\left\{\beta_{m}\right\}\right)$.

Obviously, $\psi_{c_{2} c_{1}}^{\infty}(x)=\psi(x)$ for any $x \in R^{2} \backslash \bar{D}_{1}, \phi_{c 2 c_{1}}^{\infty}(x)=\phi(x)$ for any $x \in \bar{D}_{2}$, and $\psi_{c_{1} c_{3}}^{\infty}(x)=\psi(x)$ for any $x \in D_{3}$, and we will view the mappings $\psi_{c_{2} c_{1}}^{n}, \phi_{c_{1} c_{2}}^{n} \psi_{c_{1} c_{3}}^{n}$ as approximations to the mappings $\psi, \phi$, and $\psi$, respectively. 


\subsection{Diagonalization of Translation Operators}

THEOREM 3.1. (a) For any natural $n$, each of the operators $U_{c_{2} C_{1}}^{n}, V_{c_{1 C_{2}}}^{n}, W_{c_{1} C_{3}}^{n}$, is a bounded scalar type operator (see [8, Vol. III]). Their sets of eigenvectors coincide and are given by the formula

$$
e_{q}=\left\{e^{i q m}\right\}, \quad m=0, \pm 1, \pm 2, \ldots
$$

with $q \in[0,2 \pi]$.

(b) The spectral representations of the operators $U_{c_{2} c_{1}}^{n}, V_{c_{1} c_{2}}^{n}, W_{c_{1} c_{3}}^{n}$ are respectively

$$
\begin{aligned}
U_{c_{2} c_{1}}^{n} & =\int_{0}^{2 \pi} \lambda_{n}(q) P_{q} d q, \\
V_{c_{1} c_{2}}^{n} & =\int_{0}^{2 \pi} \mu_{n}(q) P_{q} d q, \\
W_{c_{1} c_{3}}^{n} & =\int_{0}^{2 \pi} v_{n}(q) P_{p} d q,
\end{aligned}
$$

where $P_{q}$ is a rank one operator projecting $Y$ on its subspace spanned by the vector $e_{q}$ and commuting with each of the operators $U_{c_{2} c_{1}}^{n}, V_{c_{1} c_{2}}^{n}, W_{c_{1} c_{3}}^{n}$, and the functions $\lambda_{n}$, $\mu_{n}, v_{n}:[0,2 \pi] \rightarrow C^{1}$ are defined by the formulae

$$
\begin{aligned}
& \lambda_{n}(q)=\sum_{m=-n}^{n} e^{-i m\left(q+\theta_{12}\right)} J_{m}\left(k \rho_{12}\right), \\
& \mu_{n}(q)=\sum_{m=-n}^{n} e^{-i m\left(q+\theta_{12}-\pi\right)} J_{m}\left(k \rho_{12}\right), \\
& v_{n}(q)=\sum_{m=-n}^{n} e^{-i m\left(q+\theta_{13}-\pi\right)} H_{m}\left(k \rho_{13}\right) .
\end{aligned}
$$

Proof. An inspection of the formulae (3.8), (3.9), (3.10) shows that the mappings $U_{c_{2} c_{1}}^{n}, V_{c_{1} c_{2}}^{n}, W_{c_{1} c_{3}}^{n}$ are convolutions of the sequence $\left\{a_{m}\right\}, m=0, \pm 1, \pm 2, \ldots$, with the finite sequences

$$
\begin{aligned}
\left\{e^{-i m \theta_{12}} J_{m}\left(k \rho_{12}\right)\right\}, & m=0, \pm 1, \pm 2, \ldots \\
\left\{e^{-i m\left(\theta_{12}-\pi\right)} J_{m}\left(k \rho_{12}\right)\right\}, & m=0, \pm 1, \pm 2, \ldots \\
\left\{e^{-i m\left(\theta_{13}-\pi\right)} H_{m}\left(k \rho_{12}\right)\right\}, & m=0, \pm 1, \pm 2, \ldots
\end{aligned}
$$

respectively. Convolutions with finite sequences are diagonalized by the Fourier transformation (see, for example, $[8,15]$ ), which proves (a). We obtain (b) by applying the Fourier transformation to the sequences (3.26), (3.27), (3.28). 
Remark 3.3 For the mappings $U_{c_{1}{ }_{1}}^{n}, V_{c_{1} c_{2}}^{n}$, the above theorem can be extended to the case $n=\infty$. As in well known, for any $z \in C^{1}, \theta \in[0,2 \pi]$,

$$
\sum_{m=-\infty}^{+\infty} J_{m}(z) e^{i m \theta}=e^{i z \cos \theta}
$$

(see, for example, $[1,9.1 .21]$ ), and for $n=\propto$ the expressions $(3.20),(3.21)$ assume the form

$$
\begin{aligned}
& \lambda_{x x}(q)=e^{i k \rho_{12} \cos \left(q+\theta_{12}\right)} \\
& \mu_{x}(q)=e^{i k \mu_{12} \cos \left(q+\theta_{12}-\pi\right)}
\end{aligned}
$$

However, Theorem 3.1 cannot be extended to the case of the operator $W_{i ;}$ since the latter is unbounded (see [8, Vol. III]).

Renark 3.4. In numerical calculations, the operators $U_{c_{2 C_{1}}}^{n m}, V_{c_{1} c_{2}}^{n m}, W_{c_{1 C_{1}}, \text { with }}^{n m}$ sufficiently large $n, m$ will be viewed as approximations to the operators $U_{c_{2 c} c_{1}, V^{n},{ }^{n} c_{2}}$. $W_{c_{1} c_{3}}^{n}$ (see Remark 3.1). Clearly, applying either of the operators $U_{c_{2} c_{1}}^{n m}, V_{c_{1} c_{2}}^{n i n}, W_{i_{14} r_{i}}^{n+m}$ numerically to an arbitrary sequence is an order $n m$ procedure, which car be prohibitively expensive for large scale problems. However, it follows from the above theorem that the operators $U_{c_{2} C_{1}}^{n m}, V_{c_{1} c_{2}}^{n m}, V_{c_{1} c_{2}}^{n m}, W_{c_{1} c_{3}}^{n m}$ are convolutions of sequences of lengths $n, m$, and such convolutions can be evaluated in order $(n+m) \log (n+m)$ operations by means of the fast Fourier transformation (see $[4,12]$ ).

\subsection{Asymptotic Forms of Radiation Fields}

In this subsection, we introduce an alternative form of the expansions $(3.1),(3.2)$ and the mappings $U_{c_{2} c_{1}}^{n}, V_{c_{1} c_{2}}^{n}, W_{c_{1} c_{3}}^{n}$, providing a simple physical interpretation of the Lemmas 3.2-3.4 and the Theorem 3.1.

For the expansion (3.2), we will consider a function $F_{\psi, x_{0}}:[0,2 \pi] \rightarrow C^{1}$ defined by the formula

$$
F_{\psi, x_{0}}(\theta)=\lim _{t \rightarrow \infty} \psi\left(t \cdot x+x_{0}\right) \cdot \sqrt{(t)} \cdot e^{-i k t} \cdot \frac{\sqrt{(k \pi)}}{\sqrt{(2)}} \cdot e^{(\pi ; 4) i}
$$

with $x=(\cos \theta, \sin \theta)$. Substituting (2.18), (3.2), into (3.32), we obtain

$$
F_{\psi, x_{0}}(\theta)=\sum_{m=-\infty}^{+\infty} \beta_{m} e^{-(m \pi / 2)} e^{i m \theta}
$$

which provides an explicit expression of $F_{\psi, x_{0}}$ via the coefficients $\left\{\beta_{m}\right\}$, and we will refer to $F_{\psi x_{0}}$ as the asymptotic representation of the field $\psi$ with the origin at $x_{s_{3}}$.

In order to define an asymptotic representation of the field $\phi$ in $(3.1)$, we will have to introduce an additional assumption that

$$
\sum_{m=-\infty}^{+\infty}\left|\alpha_{m}\right|=c<\infty
$$


which guarantees that the expansion (3.1) converges on the whole $R^{2}$. By combining (2.17) and (3.1), it is easy to show that for any $\theta \in[0,2 \pi]$ and $x=(\cos \theta, \sin \theta)$, there exist unique numbers $V_{\theta}, V_{\theta}$ such that

$$
\lim _{t \rightarrow \infty} \psi\left(t \cdot x+x_{0}\right) \cdot \sqrt{(2 k \pi t)}-\left(e^{(k t-\pi ; 4) i} \cdot U_{H}+e^{-(k t-\pi ; 4) i} \cdot V_{\theta}\right)=0,
$$

and that the numbers $U_{\theta}, V_{\theta}$ are defined by the formulae

$$
\begin{aligned}
& U_{\theta}=\sum_{m=-\infty}^{+\infty}\left(\alpha_{m} \cdot e^{-(m \pi i 2) i}\right) \cdot e^{i m \theta}, \\
& V_{\theta}=\sum_{m=-\infty}^{+\infty}\left(\alpha_{m} \cdot e^{(m \pi, 2) i}\right) \cdot e^{i m \theta} .
\end{aligned}
$$

Now, we will define the function $G_{\phi, x_{0}}:[0,2 \pi] \rightarrow C^{1}$ by the formula

$$
G_{\phi . x_{0}}(\theta)=U_{\theta}
$$

and refer to it as the asymptotic representation of the field $\psi$ with the origin at $x_{0}$.

The following three lemmas establish that the asymptotic representations of the fields $\psi, \phi$ diagonalize the translation operators $U_{c_{2} C_{1}}^{n}, V_{c_{1} c_{2}}^{n}, W_{c_{1} c_{3}}^{n}$. All three are an immediate consequence of Lemmas 3.2-3.4 and Theorem 3.1.

LEMMA 3.5. Under the conditions of Lemma 3.2, for any $\theta \in[0,2 \pi]$,

$$
F_{\psi, c_{1}}(\theta)=F_{\psi, c_{2}}(\theta) \cdot \lambda_{\infty}(\theta)
$$

with $\lambda_{s}:[0,2 \pi] \rightarrow C^{1}$ defined by (3.30).

LEMma 3.6. Under the conditions of Lemma 3.3, for any $\theta \in[0,2 \pi]$,

$$
G_{\psi, c_{2}}(\theta)=G_{\psi, c_{1}}(\theta) \cdot \mu_{\infty}(\theta)
$$

with $\mu_{x}:[0,2 \pi] \rightarrow C^{1}$ defined by (3.31).

Lemma 3.7. Under the conditions of Lemma 3.4, for any $\varepsilon>0$ there exists $N>0$ such that for any' $n \geqslant N$,

$$
\left|\psi(x)-\phi_{n}(x)\right|<\varepsilon
$$

for any $x \in D_{3}$ where $\phi_{n}: R^{2} \rightarrow C^{1}$ is a radiation field analytical inside $D_{3}$ with the asymptotic representation defined by the formula

$$
G_{\psi_{k}, c_{3}}=v_{n}(\theta) F_{\psi, c_{1}}(\theta),
$$

and the function $v_{n}:[0,2 \pi] \rightarrow C^{1}$ is defined by (3.25). 
The following two lemmas are a direct consequence of the Theorem 3.1, Lemmas 3.5, 3.6 and the Remark 3.3. They provide explicit expressions for the asymptotic representations of the fields generated by a charge and a dipole located at an arbitrary point and for the values and the derivatives of a field given by its asymptotic representation.

LEMma 3.8. Suppose that under the conditions of the Lemma 3.2, the field is is defined by the formula $\psi=\phi_{x}^{k}$, i.e., it is the field of a wity charge located at the point . Then the asimptotic representation of the field $\psi$ with the center $c_{1}$ is given by the formula

$$
F_{\psi, c_{1}}(\theta)=e^{-i k \rho_{1} \cos \left(\theta-\theta_{1} !\right.} \text {. }
$$

If the field $\psi$ is given by the formula $\psi=\phi_{x, h}^{k}$, i.e, it is the field of a unity dipole located at the point $x$ and oriented in the direction $h$, then the asymptotic representation of (3.12) with the center at $c_{1}$ is given by the formula

$$
F_{\psi, c_{1}}(\theta)=i k \cos \left(\theta-\theta_{h}\right) e^{-i k \rho_{1} \cos \left(\theta-\theta_{1}\right)}
$$

where $\theta_{h}$ is the angle between the vector $h$ and the $x$ axis.

LEMMA 3.9. Suppose that under the conditions of the Lemma 3.3, the field $(3.13)$ has the asymptotic representation $G_{\psi \cdot c_{1}}:[0,2 \pi] \rightarrow C^{1}$. Then for any $x \in D_{1}$, he $R^{2}$ such that $\|h\|=1$,

$$
\begin{aligned}
\phi(x) & =\frac{1}{2 \pi} \int_{0}^{2 \pi} G_{\phi, c_{1}}(\theta) \cdot e^{-i k p_{1} \cos \left(\theta-\theta_{l}\right)} d \theta, \\
\left.\frac{d}{d t} \phi(x+t h)\right|_{t=0} & =\frac{i k}{2 \pi} \int_{0}^{2 \pi} G_{\phi, c_{1}}(\theta) \cos \left(\theta-\theta_{h}\right) e^{-i k p_{1} \cos \left(\theta-\theta_{!}\right)} d \theta .
\end{aligned}
$$

\subsection{Numerical Evaluation of Translation Operators}

For the rest of this paper, we will view the asymptotic representations $(3.33)$, (3.38) (as opposed to the expansions of the forms (3.1), (3.2)) as our principal tool for representing radiation fields. Lemma 3.8 permits one to calculate asymptotic representations of fields of distributions of charges and dipoles without evaluating the coefficients of their $H$-expansions, and Lemma 3.9 provides a tool for calculating the fields and derivatives of the fields with given asymptotic representations without having to evaluate the coefficients of $J$-expansions of these fields.

For a radiation field $\psi: R^{2} \rightarrow C^{1}$ analytical outside $D_{1}$ and satisfying the radiation condition (2.10), and an integer $n \geqslant 2$, we will denote by $F_{\psi \cdot c_{1}}^{n}$ the Finite sequence $a_{1}, a_{2}, \ldots, a_{n}$ defined by the formulae

$$
\begin{gathered}
a_{i}=F_{\psi, c_{i}}\left(w_{i}\right), \\
w_{i}=2 \pi \frac{(i-1)}{n}, \quad i=1,2, \ldots, n .
\end{gathered}
$$


Similarly, for a radiation field $\phi$ analytical inside $D_{1}$ and possessing and asymptotic representation $G_{\phi, c_{1}}$ we will denote by $G_{\phi, c_{1}}^{n}$ the sequence $b_{1}, b_{2}, \ldots, b_{n}$ defined by the formula

$$
b_{i}=G_{\phi, c_{1}}\left(w_{i}\right) \text {. }
$$

Obviously, $F_{\psi, c_{1}}^{n}, G_{\phi, c_{1}}^{n}$ are tabulations at $n$ equispaced nodes on the interval $[0,2 \pi]$ of the functions $F_{\psi, c_{1}}, G_{\phi, c_{1}}$, respectivcly, and we will vicw $F_{\psi, c_{1}}^{n}, G_{\phi, c_{1}}^{n}$ as finite-dimensional projections of the asymptotic representations of the $\psi, \phi$ radiation fields.

For a finite sequence $G_{\phi . c_{1}}^{n}=\left\{b_{1}, b_{2}, \ldots, b_{n}\right\}$, we will consider a radiation field $\bar{G}_{\psi, c_{1}}^{n}: R^{2} \rightarrow C^{1}$ defined by the formula

$$
\bar{G}_{\phi, c_{1}}^{n}(x)=\frac{1}{n} \sum_{j=1}^{n} b_{j} e^{-i k \rho_{1} \cos \left(w_{j}-\theta_{1}\right)}
$$

Clearly, (3.50) is a trapezoidal approximation to the integral (3.45), and we will look upon (3.50) as an approximation to the field $\phi$. Differentiating (3.50) with respect to $x$, we obtain the formula

$$
\left.\frac{d}{d t} \bar{G}_{\phi, C_{1}}^{n}(x+t h)\right|_{t=h}=\frac{i k\|h\|}{n} \sum_{j=1}^{n} b_{j} \cos \left(w_{j}-\theta_{h}\right) e^{-i k \rho_{1} \cos \left(w_{j}-\theta_{l}\right)}
$$

for any $h \in R^{2}$, and we will view (3.51) as an approximation to (3.46).

Finally, we will define mappings $P_{c_{2} c_{1}}^{m n}, Q_{c_{1} c_{2}}^{m n}, S_{c_{1} c_{3}}^{m n}: C^{n} \rightarrow C^{n}$ by the formulae

$$
\begin{aligned}
& P_{c_{2} c_{1}}^{m n}\left(z_{1}, z_{2}, \ldots, z_{n}\right)=\left(\lambda_{m}\left(w_{1}\right) \cdot z_{1}, \lambda_{m}\left(w_{2}\right) \cdot z_{2}, \ldots, \lambda_{m}\left(w_{n}\right) \cdot z_{n}\right), \\
& Q_{c_{1} c_{2}}^{m n}\left(z_{1}, z_{2}, \ldots, z_{n}\right)=\left(\mu_{m}\left(w_{1}\right) \cdot z_{1}, \mu_{m}\left(w_{2}\right) \cdot z_{2}, \ldots, \mu_{m}\left(w_{n}\right) \cdot z_{n}\right), \\
& S_{c_{1} c_{3}}^{m n}\left(z_{1}, z_{2}, \ldots, z_{n}\right)=\left(v_{m}\left(w_{1}\right) \cdot z_{1}, v_{m}\left(w_{2}\right) \cdot z_{2}, \ldots, v_{m}\left(w_{n}\right) \cdot z_{n}\right),
\end{aligned}
$$

with the functions $\lambda_{m}, \mu_{m}, v_{m}$ defined by $(3.20),(3.21),(3.22)$. It is easy to see from the formulae (3.22)-(3.23), (3.52)-(3.54) and the Theorem 3.1 that under the conditions of the Lemmas 3.2-3.4,

$$
\begin{aligned}
& P_{c^{2} c_{1}}^{m n}\left(F_{\psi, c_{2}}^{n}\right)=F_{L^{\prime}, c_{1}}^{n}, \\
& Q_{c_{1} c_{2}}^{m n}\left(G_{\psi \cdot c_{1}}^{n}\right)=G_{V \cdot c_{2}}^{n}, \\
& S_{c_{1} c_{3}}^{m n}\left(F_{\psi, c_{1}}^{n}\right)=G_{W, c_{3}}^{n},
\end{aligned}
$$

with $U=\psi_{c_{2} c_{1}}^{m}, V=\phi_{c_{1} c_{2}}^{m}, W=\bar{\psi}_{c_{1} c_{3}}^{m}$ and we will look upon the operators $P_{c_{2} c_{1}}^{m n}, Q_{c_{1} c_{2}}^{m n}$, $S_{c_{1} c_{3}}^{m n}$ as discretizations of diagonal forms of the operators $U_{c_{2} c_{1}}^{m n}, V_{c_{1} c_{2}}^{m n}, W_{c_{1} c_{3}}^{m n}$.

In order to estimate the number of nodes in the discretizations (3.47), (3.49) required to obtain a given accuracy $\varepsilon$ in the evaluation of the fields $\phi, \psi$ we will need the following well known fact (see, for example, [7]). 
LEMMA 3.10. For any integer $m$, $n$ such that $n \geqslant 2|m n|$, the n-poin trapesoldat quadrature rule on the interval $[0,2 \pi]$ integrates the function ext exactly.

Remark 3.5. By combining the above lemma with Remark 3.1, it is easy see that the number $n$ of nodes in the discretization $G_{\phi, c_{1}}^{n}$ of the function $G_{\phi, c_{1}}:[0,2 \pi] \rightarrow c^{*}$ has to be approximately equal to $2|k| R_{1}$, and is almost independent of the accuracy $\varepsilon$ with which the field $\phi$ is being calculated.

Lemmas 3.5-3.7 provide a tool for shifting the origins of asymptotic expansions of radiation fields, and for converting asymptotic representations of the form (3.32) into asymptotic representations of the form (3.38) for a cost proportional io n, where $n$ is the number of nodes in the discretization (3.48) of the interval $[0,2 \pi$. In the following two sections, this apparatus is used to construct an algorthm for rapid evaluation of integral operators of Section 2 .

\section{Rapid Evaluation of Integral Operators of Section 2}

In this section, we describe an algorithm for rapid evaluation of the field and the normal derivative of the field created on a curve $y$ by charge and dipole distributions on that same curve. For definitiveness, we will be discussing the evahation of the field created by a charge distribution. The algorithms evaluating the norrai derivative of the field created by a charge distribution, and the field and the normai derivalive of the field created by a dipole distribution are yuite similar.

\subsection{Notation}

We will consider the situation depicted in Fig. 2. The curve $\gamma$ is discretized into equispaced nodes $x_{1}, x_{2}, \ldots, x_{n}$, and we will denote the spacing between the adjacent nodes by $h$. Suppose that for each $i=1,2, \ldots, n$, a charge $a_{i}$ of strength $\sigma_{i}$

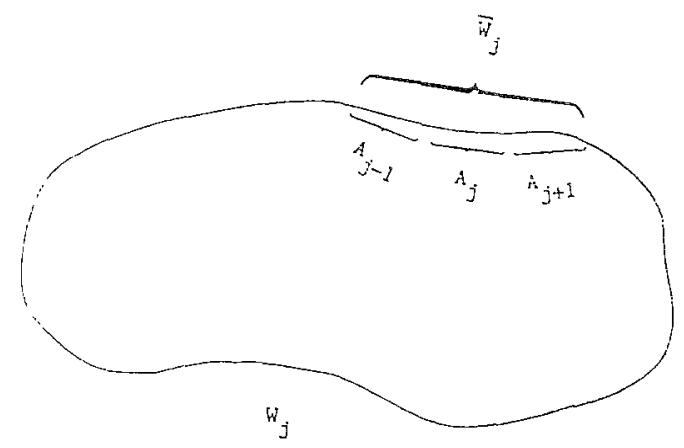

FrG. 2. Definition of the seis $A_{j}, W_{j}, W_{i}^{\prime}$. 
is located at the point $x_{i}$. In this section, we describe an algorithm for rapid calculation of approximations $g_{i}=1,2, \ldots, n$ to the sums

$$
G_{\sigma}\left(x_{i}\right)=\sum_{\substack{j=1 \\ j \neq i}}^{n} \sigma_{j} \phi_{x_{j}}^{k}\left(x_{i}\right)
$$

for $i=1,2, \ldots, n$. Clearly, this is an order $n^{2}$ process (evaluating $n$ fields at $n$ points). However, if we are interested in evaluating (4.1) with a finite accuracy (which is always the case in practical calculations), Theorem 3.1 and Lemmas 3.7, 3.8, 3.9 can be used to speed up the process.

For an integer $m \geqslant 2$, we will define the points $t_{1}, t_{2}, \ldots, t_{m+1}$ on the interval $[0, L]$ by the formula $t_{i}=(i-1) L / m$, subdividing the interval $[0, L]$ into $m$ segments of equal length and denote the center of the ith segment by $z_{i}$, so that $z_{i}=t_{i}+L /(2 m)$. For each natural $j=1,2, \ldots, m$, we will denote by $A_{j}$ the set of all charges $a_{i}$ such that $x_{i} \in \gamma\left(\left[t_{j}, t_{j+1}\right]\right)$, and $D_{j}$, the circle of radius $r=L /(2 m)$ with the center at $\gamma\left(z_{j}\right)$. We will denote by $W_{j}$ the union of all $A_{i}$ such that $\left\|z_{j}-z_{i}\right\|>3 r$, and $\bar{W}_{j}$ the union of all $A_{i}$ such that $\left\|z_{j}-z_{i}\right\| \leqslant 3 r$. Obviously. $A_{j} \subset D_{j}$ for any $j=1,2, \ldots, m$. Also, it follows from the triangle inequality that

$$
\min _{\substack{x \in A_{c} \\ y \in A_{i}}}\left\|x-y^{\prime}\right\|>r
$$

for any $i, j$ such that $A_{i} \subset W_{j}$. Finally, we will denote by $\phi_{j}$ the field of all charges $a_{i}$ such that $x_{i} \subset A_{j}$ and observe that if $x_{n} \in A_{j}$ then

$$
G_{\sigma}\left(x_{p}\right)=\sum_{A_{i} \in W_{i}} \phi_{i}\left(x_{p}\right)+\sum_{x_{t} \in W_{i}} \sigma_{i} \phi_{x_{i}}^{k}\left(x_{p}\right)
$$

\subsection{Detailed Description of an Order $n^{3 / 2}$ Algorithm}

In this subsection, $M, N$ will denote "sufficiently large" integer numbers. The actual choice of the numbers $M, N$ is discussed in the following subsection. We will evaluate the fields (4.1) in five steps.

Step 1. Using Lemma 3.8, obtain discretized asymptotic representations $F_{\left.\phi_{l}, \gamma_{-j}\right)}^{N}$ of the fields $\phi_{j}$ for all $j=1,2, \ldots, m$.

Step 2. For every pair of natural numbers $i, j \in[1, m]$ such that $A_{i} \subset W_{j}$, calculate the representation

$$
G_{\psi(j, \gamma(z))}^{N}=S_{\gamma\left(z_{i}\right), \gamma(z,)}^{M, N}\left(F_{\phi_{t}, \gamma(z),}^{N}\right)
$$

of the field $\psi_{i j}=\bar{\phi}_{\gamma\left(z_{i}\right), \gamma\left(z_{j}\right)}^{M}$ and view it as a finite-dimensional approximation to the asymptotic representation of the field $\phi_{i}$ on $D_{j}$.

Step 3. For each natural $j \in[1, m]$, calculate the sum

$$
G_{\psi_{j}, y\left(z_{j}\right)}^{N}=\sum_{A_{t} \subset w_{j}} G_{\psi_{t}, \gamma\left(z_{j}\right)}^{N}
$$


and view the field $\psi_{i}=\sum_{i} \psi_{i j}$ as an approximation to the field $\sum_{A_{1} \in W_{i}} \phi_{i}$, and $G_{i,, i t-1,}^{b}$ as a finite-dimensional approximation to the asymptotic representation of $\psi_{j}$ on $D_{j}$.

Step 4. For each natural $j \in[1, m]$, evaluate

$$
\bar{\psi}_{j}\left(x_{i}\right)=\bar{G}_{\psi, x i=j}\left(x_{i}\right)
$$

for all $i$ such that $x_{i} \in \gamma\left(\left[t_{j}, t_{j+1}\right]\right)$ and look upon (4.5) as an approximation to $\psi_{i}\left(x_{t}\right) \cdot t$.

Step 5. For each $j=1,2, \ldots, m$, evaluate the sum

$$
\tilde{\psi}_{j}\left(x_{i}\right)+\sum_{x_{p} \equiv \bar{t}_{i}} \sigma_{p} \phi_{x_{p}}^{k}\left(x_{i}\right)
$$

for all $i$ such that $x_{i} \in \gamma\left(\left[t_{j}, t_{j+1}\right]\right)$ and view (4.6) as an approximation to $G_{\sigma}\left(x_{j}\right)$.

\subsection{Choice of Parameters and CPU Time Estimate}

In the estimates below, $a, b, c, d, e$ are coefficients determined by the computer system, language, particular implementation of the aigorithm, etc.

Step 1. Obviously, this step will require order $n \cdot N$ operations (tabulating $F_{\phi_{1}, y_{i}, z_{1}}^{v}$ at $N$ nodes on the interval $[0,2 \pi]$ for each of the nodes $\left.x_{1}, x_{2}, \ldots, x_{n}\right)$. According to the Remark $3.5, N \sim|k| \cdot L / m$, and the CPU time estimate for this step becomes $a \cdot n \cdot|k| \cdot L / m$.

Step 2. For each of the pairs $i, j$ such that $A_{j} \subset W_{l}$, evaluating (4.3) will require order $N$ operations (see (3.54)), and the total number of such pairs is less than $m^{2}$, which results in the CPU time estimate of $b \cdot m^{2} \cdot n \sim b \cdot m^{2} \cdot|k| \cdot L / m=b \cdot m \cdot|k| \cdot L$ for this step.

Step 3. Obviously, evaluating the sums (4.4) for all $j-1,2, \ldots, m$ is an order $c \cdot m \cdot N \sim c \cdot m \cdot N \sim c \cdot m \cdot|k| \cdot L / m=c \cdot|k| \cdot L$ procedure.

Step 4. Evaluating (4.5) for each $i=1,2, \ldots, n$ is an order $N$ procedure, resuiting in the total CPU time estimate for this step of $d \cdot n \cdot N \sim d \cdot r_{3} \cdot|k| k \cdot L / m$.

Step 5. Evaluating the sum (4.6) for each $i=1,2, \ldots, n$ is an order $n: m$ procedure, with the resulting CPU time estimate of $e \cdot n^{2} / m$ for this step.

Summing up the time estimates for the Steps 1-5, we obtain the following time estimate for the whole process,

$$
T=A \cdot n \cdot|k| \cdot L / m+b \cdot m \cdot|k| \cdot L+c \cdot|k| \cdot L+\frac{e \cdot n^{2}}{m},
$$

with $A=a+d$, and we would like to choose $m$ in such a manner that (4.7) would 
be minimized. Differentiating (4.7) with respect to $m$, and setting the resulting derivative to zero, we obtain

$$
m_{\min }=\sqrt{\frac{A \cdot n \cdot|k| \cdot L+e \cdot n^{2}}{b \cdot|k| \cdot L}}
$$

and the corresponding minimum of (4.7) is equal to

$$
T_{\min }=2 \sqrt{A \cdot n \cdot|k| \cdot L+e \cdot n^{2}} \cdot \sqrt{b \cdot|k| \cdot L}+c \cdot|k| \cdot L .
$$

If the calculations are performed with a fixed number of nodes per wavelength (which is often a reasonable assumption), $n$ is proportional to $|k| L$, and (4.9) assumes the form

$$
T_{\min } \sim(|k| \cdot L)^{3 / 2}
$$

or

$$
T_{\min } \sim n^{3 / 2}
$$

which for large $n$ is considerably smaller than $n^{2}$.

\subsection{Further Reduction of the CPU Time Estimate of the Process}

The approach of the above subsection can be used recursively by subdividing each of the sets $A_{i}$ into subsets $\left\{B_{i j}\right\}, j=1,2, \ldots, \bar{m}$ with appropriately chosen $\bar{m}$ and representing the fields $\phi_{i}$ as sums $\phi_{t}=\sum_{t} \phi_{i j}$, where $\phi_{i j}$ is the field created by all charges $a_{p}$ such that $a_{p} \in B_{i j}$. A calculation similar to the one in the preceding section shows that such an algorithm will have an asymptotic CPU time estimate of $n^{4 / 3}$.

In [20], such a subdivision process is used recursively to obtain an order $n$ algorithm for numerical evaluation of integral operators of classical potential theory (Laplace's equation). By reproducing the construction of Section VII of [20] almost literally, one can obtain an order $n \log (n)$ algorithm for evaluating (4.1). However, our estimates indicate that for problems of practicable size $(n \leqslant 20,000)$, the improvement in actual computation times obtained by replacing an order $n^{4 / 3}$ algorithm with an order $n \log n$ algorithm would not be very significant.

\section{Implementation aNd Numerical Results}

\subsection{Implementation}

In implementing the algorithm of the present paper, we closely followed [18], as far as the formulation of the system of integral equations $(2.13),(2.14)$ and their discretization are concerned. Equations (2.13), (2.14) were discretized by means of the Nyström algorithm, which calls for replacing the integrals with finite 
quadrature formulae and the integral equations with systems of linear algebraic equations respectively (see $[3,18]$ ). Since the kernels of the equations $\{2,13\}$, 12.14) are logarithmically singular, quadrature formulae suitable for functions with logarithmic singularities have to be employed, and we used a fourth-onder convergent formula of the type discussed in [21]). Discretization of Eqs. (2.13), (2.14) by means of the Nyström algorithm leads to large scale, asymptotically wellconditioned systems of linear algebraic equations (see [3]), to which we apply a version of the generalized conjugate residual algorithm (see [11]). Solution of a linear system by means of a generalized conjugate residual-type algorithm involves application of the matrix of the system to a sequence of recursively generated vectors, which is accomplished by means of the algorithm of the preceding section (we implemented an order $n^{4 \cdot 3}$ version of the process). As is well known, in order to converge to a relative accuracy $\varepsilon$, a generalized conjugate residual-type algorithm requires order $-K \cdot \log (\varepsilon)$ iterations, where $K$ is the condition number of the matrix of the system being solved. This results in the total CPU time estimate of the order $-n^{43} \cdot \log (\varepsilon)$ for the solution of the Eqs. $(2.13),(2.14)$, since the application of the Nyström algorithm to these equations leads to weil-conditioned linear sysiems (see [3]). After the system of Eqs. (2.13), (2.14) is solved, evaluating the scattered field at any point $x \notin \Gamma$ involves two numerical integrations over $\Gamma$ levaluating the field of the charge distribution and that of the dipole distribution/, which is an ordes $n$ procedure. This brings us to the estimate

$$
T_{\mathrm{SOLVE}}=-a \cdot \log (\varepsilon) \cdot n^{4}+b \cdot n \cdot m
$$

for obtaining the solution of the problem (2.6)-(2.10), where $m$ is the number of points in $R^{2}$ where the solution has to be calculated.

\subsection{Numerical Results}

We have applied the algorithm of the present paper to several acoustic scattering problems in two dimensions. In the three examples presented below, all calculations were performed in single precision on a VAX-8600 computer. and all timings are reported in seconds of CPU time on that machine.

EXAMPLE a. Scattering from a circular inclusion. In this case, the problem possesses an analytical solution, providing a convenient way to verify the accuracy of the algorithm. In our example, the radius of the scatterer was $100 \mathrm{ft}$, ard its center was at the origin. The densities inside and outside the scatterer were 1.2 and 1.0, respectively, and the speeds of sound were 20 and 18 , respectively. The ambient field was generated by a cylindrically symmetric source located at the point $(100,100)$, and the scattered field obtained by the algorithm of the present paper was compared to the analytical result at 40 equispaced points located on the circle of radius 110 with the center at the origin (see Fig. 3). The calculation was performed with the angular frequency of the source varying from 10 to 320 , and in all cases the boundary of the scatterer was discretized at 10 nodes per wavelength, which usually results in three to four digit accuracy. In Table I, $w$ is the angular 


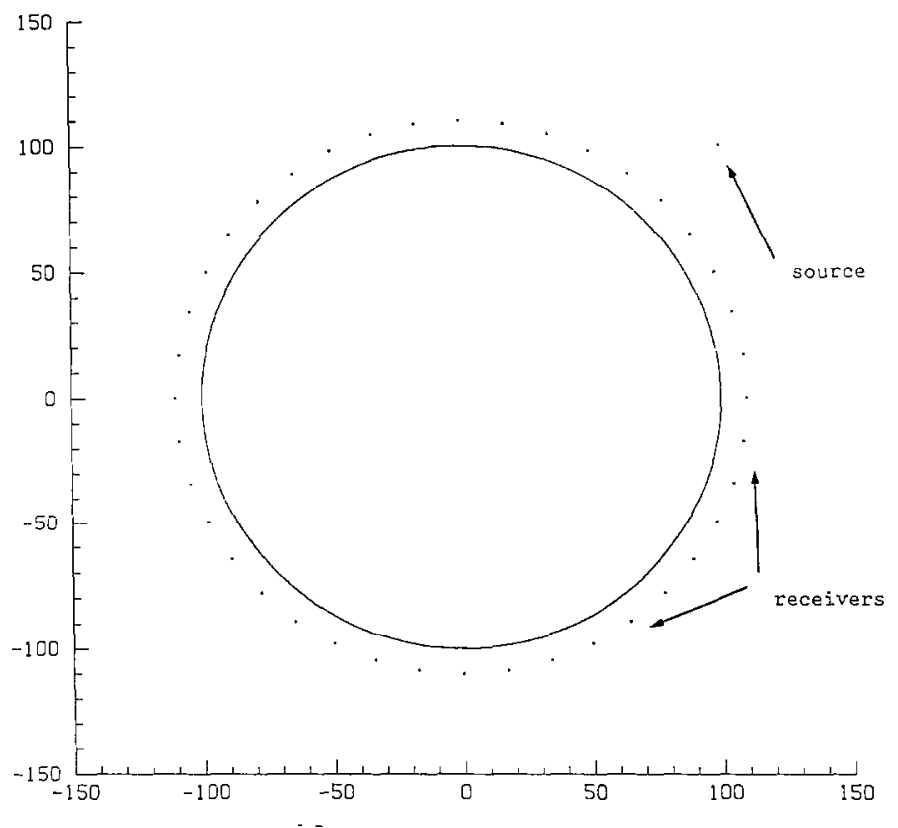

FIG. 3. Acoustic scattering from a circular inclusion.

frequency, $n$ is the number of nodes on the boundary of the scalterer (at 10 nodes per wavelength), $\varepsilon$ is the resulting mean square error at 40 receivers, and $m$ is the number of iterations the generalized conjugate residual algorithm (GCRA) took to converge to 4-digit accuracy. Following observations can be made from Table I.

1. The accuracy of the solution obtained with a given number of nodes per wavelength is virtually independent of the frequency.

2. The number of iterations required by the GCRA to obtain a given accuracy is almost independent of the frequency.

TABI E I

CPU Times and Accuracies for the Example a

\begin{tabular}{rrrrr}
\hline$\omega$ & $n$ & $=$ & $m$ & $T(s)$ \\
\hline 10 & 128 & $0.766 \mathrm{E}-3$ & 5 & 36 \\
20 & 257 & $0.594 \mathrm{E}-3$ & 6 & 83 \\
40 & 514 & $0.629 \mathrm{E}-3$ & 6 & 179 \\
80 & 1028 & $0.620 \mathrm{E}-3$ & 6 & 612 \\
160 & 2056 & $0.708 \mathrm{E}-3$ & 6 & 1696 \\
320 & 4112 & $0.727 \mathrm{E}-3$ & 6 & 4328 \\
640 & 8224 & $0.693 \mathrm{E}-3$ & 6 & 10.5017 \\
\hline
\end{tabular}


3. The actual CPU times required by the algorithm grow somewhat eraticaligy as a function of the number of nodes on the boundary, but seem to be in agreement with the theoretical CPU time estimate of the algorithm.

EXAMPLE $b$. Scattering from a lense-shaped object. In the situation depicted in Fig. 4, the speed of sound in the containing space is $30 \mathrm{ft} / \mathrm{s}$, and the speed of sound inside the scatterer is $20 \mathrm{ft} / \mathrm{s}$. Both upper and lower surfaces of the scatterer are circular with the radius of curvature $1 \mathrm{ft}$, so that the focal distance of the resuiting lence is $2 \mathrm{ft}$. Three cylindrically symmetric sources are located at the points $I$. $I$, and III respectively have the frequency $F=7162 \mathrm{~Hz}$, resulting in the wavelengts $0.0041888 \mathrm{ft}$ outside the scatterer, and $0.0027925 \mathrm{ft}$ inside the scatterer. The amplitude of the field generated by this configuration on the screen is depicted in Fig. 5. Clearly, the laws of geometrical optics should be applicable to this situation with a reasonable degree of accuracy, since the lense is about 240 exterior for 360 interior) wavelengths in diameter, and a careful examination of the Fig. 5 shows this to be the case. In order to produce Fig. 5, the surface of the lense in Fig. 4 was discretized into 6134 nodes (with 10 nodes per wavelength), resulting in a system of linear algebraic equations of dimension 12268. The solution of this problem with a two-digit accuracy took $3358 \mathrm{~s}$ of CPU time on a VAX-8600.

EXAMPLE c. Radiation from a worm-shaped object. In this numerical experiment, a source of angular frequency $\omega=640$ was located inside an inclusion of a

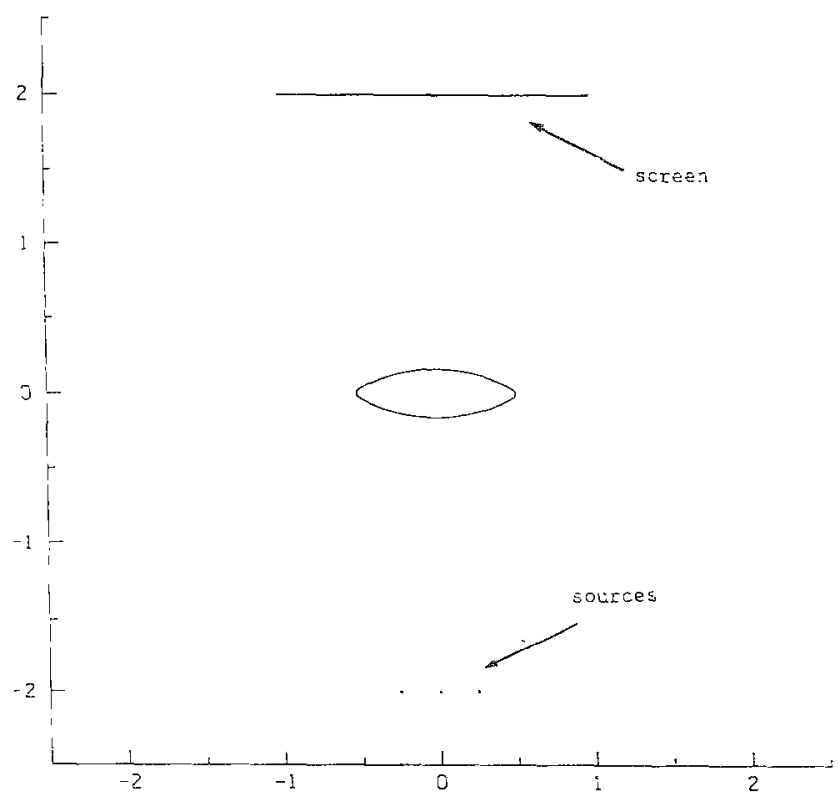

FIG. 4. Acoustic scattering from a lense-shaped inciusion. 


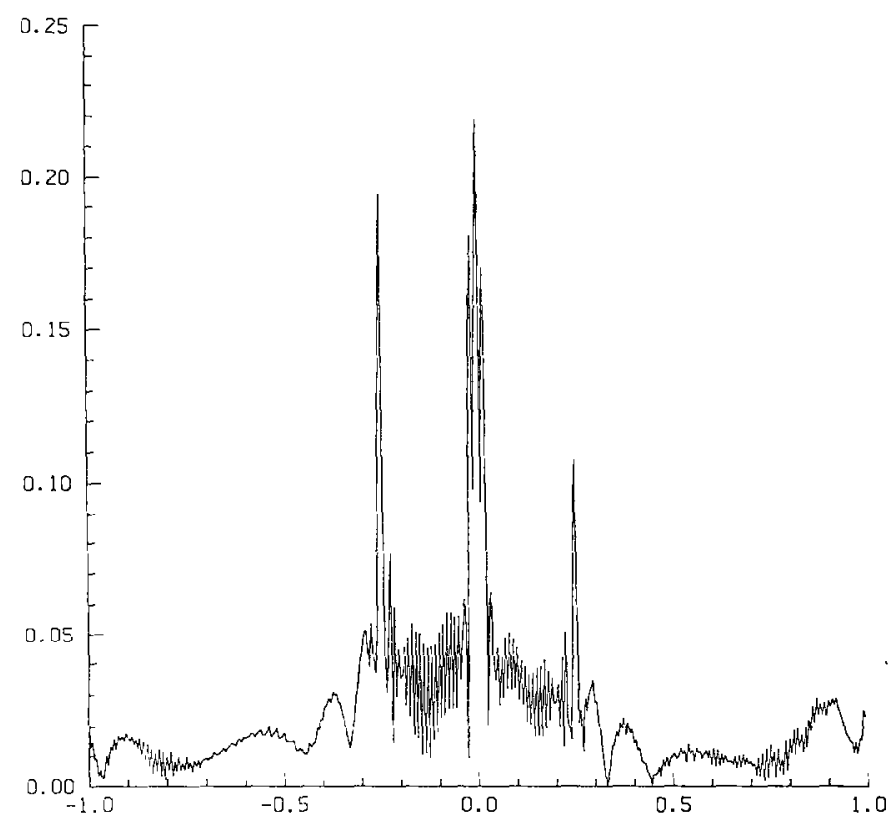

FIG. 5. Amplitude of the field measured along the screen in Fig. 4.

complicated shape in a fluid two-dimensional space (see Fig. 6). The densities inside and outside the inclusion were 1.25 and 1.0 , respectively, and the speeds of sound were 20 and 25 , respectively. The boundary of the inclusion was discretized at 10 nodes per wavelength, resulting in the total number of 5078 nodes on the boundary of the inclusion. The calculation took 3867 seconds of CPU time to obtain the solution with two-digit accuracy, and the amplitude of the field as measured by receivers in Fig. 6 is displayed in Fig. 7.

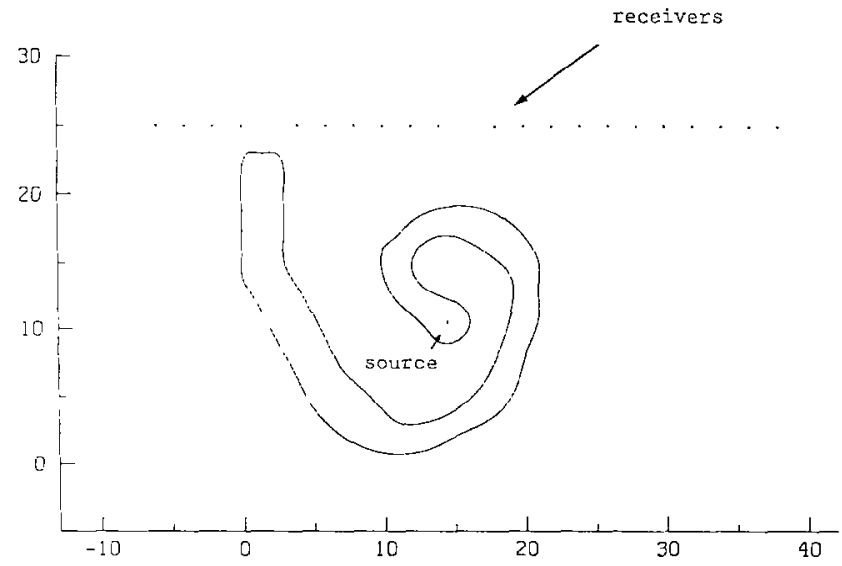

Fig. 6. Acoustic radiation from a worm-shaped inclusion. 


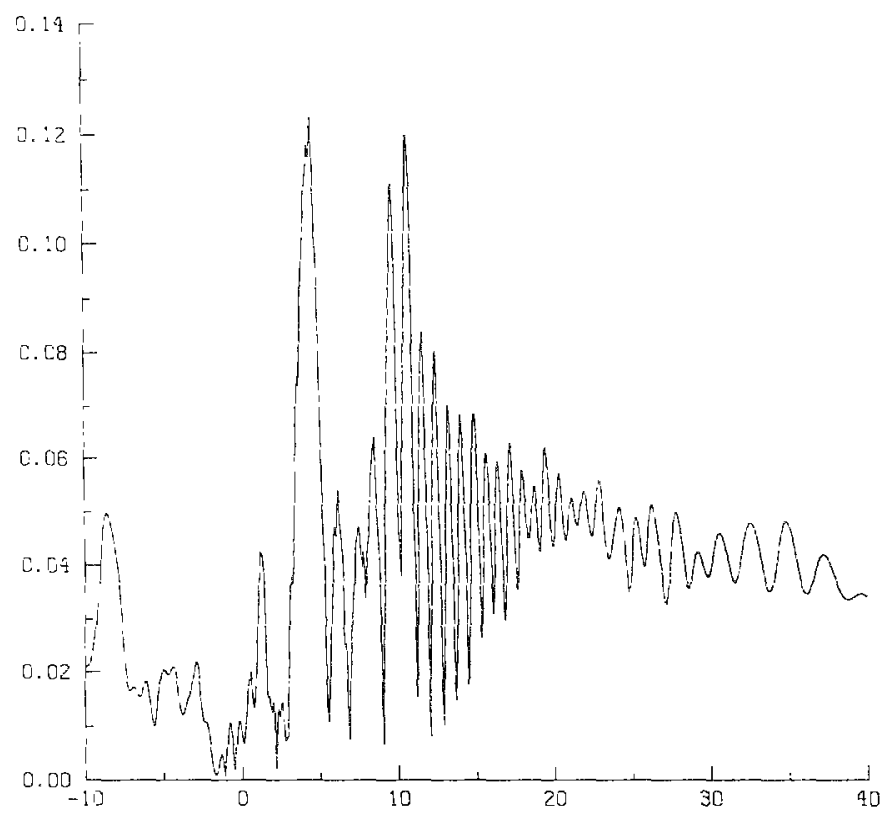

FiG. 7. Amplitude of the field measured by receivers ir. Fis. 6.

Remark 5.1. An alternative approach to the solution of problems of types b-c would be to impose a finite difference grid on the region containing the scatterer. impose some form of absorbing boundary conditions (see, for example, [12]) at the boundary of the discretized region, and attempt to solve the resulting systeri of linear algebraic equations iteratively. At 10 nodes per wavelength, the grid in Examples b, c, would be at lcast of the size $4000 \times 4000$ nodes, and the condition number of the resulting system would be of the order $(4000)^{2} \sim 10^{7}$. Naturally, as: 0 nodes per wavelenth, the sharp boundary of the scatterer would be resoived quite poorly, yielding an accuracy of the solution of perhaps one digit. Even ignoring the difficulties associated with the quality of existing non-reflecting boundary condinion: the problem is, clearly, unmanageable.

\section{Generalizations and Conclusions}

\subsection{Other Boundary Conditions}

In the present paper, we have been solving the problem (2.6) 12.10), corresponding to the scattering of sound from a fluid inclusion in a fluid spase. Obviously, the two classical acoustical scattering problems (Dirichlet and Neumann problems for the Helmholtz equation, corresponding to scattering from a cavity and from a rigid inclusion respectively) can be solved in a similar manner. 
Generally, whenever a scattering problem is reduced to a set of second kind integral equations on the boundary of the scatterer by means of some form of Stoke's theorem, the algorithm of Section 4 provides a tool for solving these equations in order $n^{4 / 3}$ operations.

\subsection{Multiple Scatterers}

The case of multiple scatterers does not differ substantially from the case of a single scatterer. The scattered field inside each scatterer is represented in a manner precisely similar to the one used to solve a single scatterer problem. The scattered field outside the scatterers is represented by the sum of the fields of single and double layer potentials on the surfaces of all scatterers. The whole procedure is completely straightforward.

\subsection{Three-Dimensional Version of the Theory}

Three-dimensional equivalents of the expansions (3.1), (3.2) are well known (see, for example, [16]), and those of the mappings $U_{c_{2} c_{1}}^{n}, V_{c_{1} c_{2}}^{n}, W_{c_{1} c_{3}}^{n}$ are fairly easy to define. However, in two dimensions, the translation operators $U_{c_{2} C_{1}}^{n}, V_{c_{1} c_{2}}^{n}, W_{c_{1} C_{3}}^{n}$ are convolutions, and the diagonal form of the latter is well known from the standard theory of the Fourier integral (see Theorem 3.1). In three dimensions, no such ready-made analytical apparatus is available, and the proof of an equivalent of the Theorem 3.1 is considerably more involved. Furtunately, diagonal forms of the three-dimensional analogues of the operators $U_{c_{2} C_{1}}^{n}, V_{c_{1} C_{2}}^{n}, W_{c_{1} c_{3}}^{n}$ can be obtained by a generalization of the technique used in [22] to derive Graf's addition theorem, permitting fast solvers for scattering problems in three dimensions to be constructed. Details of this generalization will be reported at a later date.

\subsection{Conclusions}

One of principal difficulties arising in the solution of large-scale scattering problems of integral equations is the fact that the Green's function for the Helmholtz equation decays slowly. As a result, the kernels of the obtained integral equations are not sparse, and their discretization leads to dense large-scale systems of linear algebraic equations. Solution of such systems is an extremely expensive proccss (see $[9,11]$ ), which limits the usefulness of the whole approach.

In Section 4 of the present paper, we construct an algorithm for rapid application of the matrices resulting from discretization of integral equations of scattering theory to arbitrary vectors. The asymptotic CPU time estimate of the algorithm is $n^{4 / 3}$ and can be reduced to $n \log (n)$, where $n$ is the number of nodes in the discretization of the boundary of the scatterer. By combining the approach of the Section 4 with a generalized conjugate residual type process, we obtain an order $-n^{4,3} \log (\varepsilon)$ algorithm for the solution of the integral equations $(2.13),(2.14)$ of acoustic scattcring thcory. This results in acceptable computation times even for large-scale scattering problems (see preceding section), as long as the solution has to be evaluated at a limited number of points. 


\section{REFERENCES}

1. M. Agramowitz and 1. Stegun, Handbook of Mathematicni Funciions, Applied Math. Saries (National Bureau of Standards, Washington, DC. (1964).

2. J. D. AcheNBaCH, Wave Propagation in Elastic Solids (North-Holland, New York, 1980 ).

3. K. E AtKInson, Fredholm Integral Equations of the Second Kind (Siam. Philadelphia, 1976).

4. E. O. Brigham. The Fast Fourier Transform (Prentice-Hall. Englewood Cliffs. NJ, 1974).

5. D. COLton AND R. KREss, Integral Equations Methods in Scattering Theory (Wiley, New York. 1983\}.

6. R. Courint and D. Hilbert. Methods of Mathematical Physics (Interscience, New York. 1966).

7. G. Deinlquist and A. Brork, Numerical Methods (Prentice-Hall, Englewood Ciffs, NJ, 1974$).$

8. N. DUNFORD AND J. T. SCHWARTZ, Linear Operators (Interscience. New York, 1971).

9. I. S. DUF, Recent developments in the solution of large sparse linear equations. in Proceedings, INRLA Fourth International Symposium on Computing Methods in Applied Science and Engineering. Fersailles, 1979. edited by I. S. Duff, (Springer-Verlag, Ncw York Berlin, 1979).

10. J. W. Dunkin, Bull. Seismol. Soc. Amer. 55, 335 (1965).

11. S. C. Eisenstat, H. C. Elman, and M. H. Schultz, SiAM J. Numl. Anal. 20, 345 (1983).

12. D. F Elliott and K. R. RaO, Fast Transforms (Academic Press., New York, 1982)

13. B. Engquist and A. Majda, Math. Comput. 31, 139 (1973).

14. A. C. Eringen and E. S Suhubr, Elastodinamics (Academic Press, New York, 1975).

15. T. Kato, Perturbation Theory for Linear Operators (Springer Verlag, New York. 1976).

16. N. S. Koshliakov, M. M. Smirnov. and E. B Guner, Differential Equations of Mathematical Physics (North-Holland, Amsterdam, 1964).

17. R. KRESS AND G. F. ROACH, J. Math. Phys. 19, 1433 (1978).

18. V. RoKHLIN, Wave Motion 5, 257 (1983).

19. V. RokHLIN, Comput. Math. Appl. 11, 667 (1985).

20. V. RokHLin. J. Comput. Phys. 60, 1987 (1985).

21. V. RokhuIN, Technical Report 441, Dept. of Computer Science, Yale University, 1:y5 (unpublished).

22. G. N. Watson. A Treatise on The Theory of Bessel Functions (Cambridge Univ. Press, Cambridge, 1980:

23. R. WINTER. SIAM J. Numer. Anal. 17. 14 (1980). 